\title{
APROPIACIÓN DE LOS BIENES COMUNES DE LA NATURALEZA EN ARGENTINA: ENTRE LA GESTIÓN AMBIENTAL Y LOS TERRITORIOS DE VIDA ${ }^{1}$
}

\begin{abstract}
Pablo Barbetta ${ }^{2}$
Diego Domínguez ${ }^{3}$

Resumen

La incorporación de la cuestión ambiental en las agendas de gobierno ha promovido la intensificación de la gestión y de la privatización de los bienes de la naturaleza. El presente artículo tiene por objetivo analizar diversos dispositivos de gestión ambiental problematizando tales modalidades a la luz de la hipótesis que señala el ascenso de un devenir posible de la cuestión ambiental como desarticulación del dominio público y de las formas consuetudinarias de significación/apropiación de la naturaleza.

Palabras clave: cuestión ambiental; gobernanza ambiental; apropiación; privatización; mercantilización.
\end{abstract}

\section{APROPRIAÇÃO DE BENS COMUNS NA ARGENTINA: ENTRE GESTÃO AMBIENTAL E TERRITÓRIOS DA VIDA.}

\section{Resumo}

A incorporação da questão ambiental nas agendas governamentais promoveu a intensificação da gestão e privatização dos bens comuns. O objetivo deste artigo é analisar vários dispositivos de gestão ambiental, problematizando tais modalidades à luz da hipótese que indica o surgimento de um futuro possível da questão ambiental, como desarticulação do domínio público e formas costumeiras de significância / apropriação de a natureza.

Palavras-chave: questão ambiental; governança ambiental; apropriação; privatização; mercantilização.

\section{APPROPRIATION OF COMMON ASSETS OF NATURE IN ARGENTINA: BETWEEN ENVIRONMENTAL MANAGEMENT AND THE TERRITORIES OF LIFE}

\begin{abstract}
The incorporation of the environmental issue in government agendas has promoted the intensification of the management and privatization of natural assets. The purpose of this article is to analyze various environmental management devices by problematizing such modalities in the light of the hypothesis that indicates the rise of a possible future of the environmental issue as disarticulation of the public domain and customary forms of significance / appropriation of nature.
\end{abstract}

Keywords: environmental issue; environmental governance; appropriation; privatization; commodification.

1 Artigo recebido em 10/11/2019. Primeira avaliação em 03/02/2020. Segunda avaliação em 12/02/2020. Aprovado em 19/02/2020. Publicado em 22/05/2020.

DOI: https://doi.org/10.22409/tn.v18i36.38538

${ }^{2}$ Universidad de Buenos Aires, Facultad de Ciencias Sociales, Instituto de Investigaciones Gino Germani, Consejo Nacional de Investigaciones Científicas y Técnicas (CONICET), Buenos Aires Argentina. E-mail: pbarbetta@sociales.uba.ar. https://www.conicet.gov.ar/new_scp/detalle.php?id=26961\&datos_academicos=yes. ORCID: 0000-0001-8032-3923

${ }^{3}$ Universidad de Buenos Aires, Facultad de Ciencias Sociales, Instituto de Investigaciones Gino Germani, Consejo Nacional de Investigaciones Científicas y Técnicas (CONICET), Buenos Aires Argentina. E-mail: https://www.conicet.gov.ar/new_scp/detalle.php?id=30241\&datos_academicos=yes ORCID: 0000-0002-9434-4677 


\section{Introducción}

En el origen de las sociedades modernas capitalistas está la apropiación privada de la tierra (suelo, subsuelo, agua, mares y bosques) y su transmutación en mercancía. Por la vía de la violencia o de la compra y venta en el mercado, la tierra se transmutó en propiedad y en mercancía. Esta es la forma moderna capitalista que asumió la relación sociedad-naturaleza. Una relación de control y "dominio" de la naturaleza basada en la apropiación privada de la tierra (POLANYI, 1989). Ahora bien, en la actualidad y debido a la mediación de los avances tecno-científicos asistimos a un proceso de sobre mercantilización de la naturaleza (LEFF, 2005), basado en la privatización de la biodiversidad. De modo tal, aquella primera ruptura del metabolismo social (MARX, 1997), con la separación del productor directo de la tierra y su "laboratorio natural", hoy es reeditada o profundizada en nuevos términos cuyo alcance apenas avizoramos, gestando nuevas modalidades de intervención humana en los ciclos de vida, nuevas formas jurídicas para el derecho de propiedad, nuevas modalidades de control territorial, e incluso lo que algunos autores denominan como nuevas formas de apropiación económica tal como la "renta de la vida" (BARTRA, 2008).

Paralelamente a estos procesos, la cuestión ambiental está íntimamente vinculada a la crisis ecológica como consecuencia de esta nueva etapa del desarrollo de la modernidad capitalista o del bio-capitalismo, que nace con el control genético de la vida y la administración del ADN como recurso. Las nuevas tecnologías asociadas con este proceso son: biotecnología, nanotecnología, electroinformática, nuevas energías (DELGADO RAMOS, 2015) y cuyas manifestaciones más palpables son el cambio climático, la desertificación, la pérdida de biodiversidad, alteración de los ciclos biológicos, erosión genética, etc.

En este contexto, la cuestión ambiental, en pocas décadas, cristalizó un conjunto vasto de regulaciones con escala local, nacional y global, tendientes a enfrentar lo que se ha definido como "crisis ecológica" (BARBETTA, COX, DOMINGUEZ Y PESSOA, 2018). Este despliegue se erige como conceptos clave y hoja de ruta en la escala global la perspectiva del desarrollo sustentable a partir de la década de 1990, y de la economía verde desde finales de la década del 2000.

Sin embargo, tanto el diagnostico en términos de crisis ambiental, como las respuestas de intervención local y global, se han inscripto dentro del arte neoliberal 
de gobierno (SEOANE, 2017) reformulando los pilares de la modernidad occidental en términos ecológicos. En efecto, entendemos que la cuestión ambiental, en clave de arte neoliberal de gobierno, opera en base a un conjunto de dispositivos para la promoción de la privatización y mercantilización de la naturaleza. Estos dispositivos pueden ser integrados bajo la figura genérica de la gobernanza ambiental en la escala nacional y global: áreas protegidas, ordenamientos territoriales, corredores biológicos, etc. Sin embargo, a la par de dichos dispositivos y/o regulaciones podemos observar la ampliación de la conflictualidad entre apropiaciones y valoración de los bienes naturales e inmateriales, sobre todo, por campesinos y pueblos originarios y poblaciones rururbanas.

De modo tal, el presente documento toma como punto de partida la gobernanza ambiental como intento de salida o administración de la crisis ecológica. A partir de ello se analizan diversos dispositivos o modalidades de gestión ambiental: ordenamientos territoriales, corredores de biológicos y áreas protegidas, entre otros. Finalmente se problematizaran tales modalidades a la luz de la hipótesis que señala el ascenso de un devenir posible de la cuestión ambiental como desarticulación del dominio público y de las formas consuetudinarias de significación/apropiación de la naturaleza. El análisis de las modalidades de gobernanza ambiental ha mostrado que a pesar de establecer derechos de tercera generación, y referir al interés general, intensifican el dominio privado del mundo a partir de la mercantilización y artefactualización de la vida.

\section{Regulación y emancipación en un contexto de gobernanza ambiental}

Dentro de la teoría social contemporánea es cada vez más frecuente el planteo que sostiene que nos encontramos en un periodo de transición paradigmática, siendo éste un proceso que no sólo se refiere a una transición epistemológica, sino que implica también una transformación del paradigma societal. Desde dicha perspectiva las sucesivas crisis de la sociedad capitalista contemporánea muestran las deficiencias del paradigma dominante, ya que ponen de manifiesto que éste ya no es capaz de explicar o dar respuestas a los reclamos y expectativas de gran parte de la ciudadanía (SANTOS, 2000). Dicha transición supuso que la tensión entre el pilar de la regulación y el pilar de la emancipación, pilares en los que descansa el paradigma de la modernidad, entrara en un largo proceso histórico de degradación caracterizado 
por la gradual y creciente transformación de las energías emancipatorias en energías regulatorias como consecuencia de "la reducción de la emancipación moderna a la racionalidad cognitivo-instrumental de la ciencia y la reducción de la regulación moderna al principio del mercado, alimentado por la conversión de la ciencia en la fuerza primordial de producción" (SANTOS, 2009, p. 40). Pero el colapso de la emancipación en la regulación también supone el concurso del derecho moderno. Si entendemos al derecho, es decir, las leyes, las normas, las costumbres, las instituciones jurídicas, como un conjunto de representaciones sociales, la subordinación de su lógica a la racionalidad cognitivo -instrumental de la ciencia moderna también implicó que éste entrara en crisis.

En este contexto, si la emancipación se vuelve impensable, la regulación se vuelve imposible como consecuencia de una nueva sensación de inseguridad provocada por la posibilidad real de desarrollos incontrolables basada en la creciente asimetría que se da entre la capacidad de obrar y la capacidad de prever (SANTOS, 2009). Ejemplo de esto para el tema que nos compete es que, por un lado, idea de la naturaleza como un mecanismo que puede ser controlado y dominado a partir del descubrimiento de las leyes que la gobiernan bajo el supuesto beneficio común de la humanidad, ha generado la explotación excesiva e insensata de los bienes naturales con consecuencias impredecibles. Por el otro, la primacía del principio de mercado conlleva a la mercantilización y apropiación privada de los bienes naturales a partir tanto de dispositivos legales como ilegales.

Si bien autores como Boaventura de Sousa Santos realiza una profunda crítica a la idea de la ciencia, en general, y a la idea del progreso científico en particular, la salida al manejo de los excesos e insuficiencias de la modernidad ha sido desde el estado como desde mercado, exigir más progreso científico y la profundización de la naturaleza como artefacto. En este contexto, se ha desarrollado lo que algunos autores denominan como el "arte neoliberal de gobierno". Se entiende por esto último, retomando algunos elementos de Foucault, "el conjunto diverso de racionalidades, tecnologías, prácticas discursivas y extradiscursivas orientadas a modelar y producir las conductas de individuos y poblaciones" (SEOANE, 2017, p. 80) que incluye la acción del Estado pero que también lo trasciende en otras formas institucionales y realidades más amplias caracterizadas por el predominio de la libertad de mercado como principio que orienta todo tipo de intervención. En este punto podemos afirmar 
que mientras el modelo neoliberal ha llevado a la apropiación privada de la naturaleza incorporándola al mercado, el campo académico que trata el problema ambiental reconoce que la racionalidad económica neoliberal no pone atención a la degradación ambiental y sí a su propia reproducción económica vinculada a su capacidad de innovación en la resolución de problemas ambientales y la internalización de ciertos costos (O'Connor 2002). En este contexto, la lógica neoliberal propone una subordinación de la naturaleza por la economía, del espacio vital por el espacio económico global, mediante un proceso de mercantilización o capitalización de la naturaleza, que, a partir de la crisis ambiental, debe inscribirse en una lógica "sustentable" (BARBETTA, DOMíNGUEZ, COX Y PESSOA, 2018). Así, desarrollos teórico -políticos como la economía ambiental (desarrollo sustentable), la ecología de mercado (economía verde o capitalismo verde) y la ecología económica pretendieron y en parte 4 lograron rehacer la naturaleza a "imagen y semejanza" del capital (O'CONNOR, 2002). Es la recreación de una naturaleza plenamente capitalista, es la gestación de una segunda naturaleza, en tanto (re)producida como mercancía (valor de cambio), y a su vez, en tanto objeto de conocimiento administrable y gestionable como artefacto (O'CONNOR, 2002).

En este contexto, frente a la desacreditación de la regulación como pilar de la modernidad, la gobernanza se ha erigido como propuesta para la solución de los dilemas regulatorios planteados que tanto los mercados como los Estados no pueden solucionar por sí mismos (SANTOS Y RODRÍGUEZ GARAVITO, 2007). Sin embargo, debemos destacar que, por un lado, el enfoque de la gobernanza tiende a ignorar las profundas asimetrías de poder entre actores y ve la esfera pública como un espacio despolitizado de colaboración entre «actores» genéricos (SANTOS Y RODRÍGUEZ GARAVITO, 2007). Por el otro, la gobernanza como intento de reconstruir la importancia de la regulación social pero también de la emancipación social en base a la participación y el conocimiento local de las poblaciones, se da en un contexto de crisis del contrato social (SANTOS, 2006).

Con la crisis del estado de bienestar y el paso al periodo neoliberal, "la lógica de la apropiación/violencia ha ido ganando fuerza en detrimento de la lógica de

\footnotetext{
${ }^{4}$ Armando Batra (2008) sostiene que si bien algunos de los elementos constitutivos de la naturaleza puede ser privatizables e intervenidos por el capital, éste fracasa en el intento de subordinarla totalmente por sus imperativos en la medida en que la ciencia moderna no puede descifrar completamente las leyes que gobiernan a la naturaleza.
} 
regulación/emancipación” (SANTOS, 2006, p.38). Incluso siguiendo el argumento del autor podríamos decir que "el dominio de la regulación/emancipación no sólo está contrayéndose sino contaminándose internamente por la lógica de la apropiación/violencia" (SANTOS 2006, p. 38). Por apropiación el autor entiende la incorporación, la cooptación y la asimilación, mientras que la violencia conlleva la destrucción física, material, cultural y humana (SANTOS, 2006). Una y otra operan profundamente entrelazadas y de forma disímil de acuerdo a los contextos socioeconómicos y políticos.

En este contexto, la gobernanza ambiental en tanto discurso para superar la crisis ambiental se pone de manifiesto en un sinfín de regulaciones internacionales, regionales y nacionales con el objetivo de ordenar la actividad económica y sus impactos sobre el medio ambiente, legislaciones que si bien regulan, muchas veces lo hacen a partir de dispositivos que suponen el despliegue de una lógica tendiente a la apropiación y/o a la violencia. Estos dispositivos pueden ser integrados bajo la figura genérica de la gobernanza ambiental en la escala nacional y global: servicios ambientales, bonos verdes, cambios de uso de suelo, aprovechamiento sustentable de los bienes naturales, energías limpias y renovables, etc.

La gobernanza ambiental repone la propuesta del desarrollo como aprovechamiento sustentable de los recursos naturales. Las principales iniciativas económicas de Latinoamérica y el Caribe, con mayor o menor planificación estatal, buscan inscribirse en este marco. En la escala sudamericana se destaca en este sentido la "Iniciativa para la Integración de la Infraestructura Regional Suramericana" (IIRSA). Se trata de la planificación gubernamental de las condiciones físicas para la explotación de recursos naturales de los países de la región, a partir de proyectos en las áreas de "transporte, energía y comunicación". Con diferentes grados de avance, los gobiernos de los países integrados han consensuado (y ejecutan) más de 500 proyectos en estos tres sectores. Según los análisis críticos el IIRSA "busca mejorar la conectividad del 'corazón' de la América del Sur con el objetivo de mantener el modelo primario exportador", de modo tal que "no sólo es la presión sobre la naturaleza sino también la exclusión que estos megaproyectos generan en las poblaciones locales" (DE LISIO, 2013). Sin embargo, según los postulados del IIRSA "la integración y la unión suramericanas se basan en principios como participación ciudadana y pluralismo, y en la reducción de las asimetrías y armonía con la 
naturaleza para alcanzar el desarrollo sostenible" (http://www.iirsa.org/). A pesar de las promesas de "progreso" y de "desarrollo sostenible" con "participación ciudadana" para las economías de la región, una parte significativa de los movimientos campesinos, indígenas y ambientalistas, de base local, han puesto en duda tales argumentos, cuestionado esta iniciativa dado su carácter "extractivista".

Como contraparte o complemento existen iniciativas de integración ecológica para la conservación de los recursos naturales, principalmente la biodiversidad. Son los corredores biológicos, como conectividad de flora y fauna, a lo largo de regiones y continentes. En Latinoamérica un caso destacado es la creación del Corredor Biológico Mesoamericano (CBM), que cubre el 7\% de la biodiversidad planetaria en más de 100 millones de hectáreas comprendidas entre Belice, Costa Rica, El Salvador, Honduras, México, Nicaragua y Panamá (CCAD, 2005). Esta modalidad de conservación recibe fondos muy importantes de centenas de millones de dólares del Banco Mundial y del Banco Interamericano de Desarrollo, por ser considerado estratégico a nivel geopolítico de la región. En este sentido surge de los documentos oficiales el segundo propósito ligado con la conservación de la biodiversidad, es decir, su valorización económica. Según el modelo win-win, la protección de la biodiversidad es posible a través de la bioprospección: "la exploración de la biodiversidad para encontrar recursos comercialmente valiosos para la genética y la bioquímica" (BOZA, 1994, citado por DELGADO RAMOS, 2015).

Un problema en aumento relacionado con la gobernanza ambiental de los territorios, es la creación y funcionamiento de las áreas protegidas, sean estatales o administradas por filántropos, ONGs o corporaciones multinacionales. Se trata de una nueva cuestión que reactualiza antiguas problemáticas. La conservación de ecosistemas y biodiversidad, a partir de estas figuras, se ha reforzado sin que se resuelva desde el Estado la actitud hacia las comunidades y poblaciones locales que habitan dentro de esas áreas: campesinos, pescadores, indígenas, etc. Si por un lado se crean nuevas áreas protegidas ligadas a la economía verde de los bonos de carbono o bien para la recuperación/investigación de fauna y flora, turismo sustentable, etc. Por otro lado, se desconocen los derechos de poblaciones ancestrales a reproducir sus modos de vida (caza, recolección, pesca, agricultura, ganadería), y son perseguidas por fuerzas de seguridad o policías ambientales por su carácter de "depredadores", "productores insustentable", "contaminadores", 
"traficantes de madera", "intrusos", "cultivadores ilícitos", etc. Algunos ejemplos de estos fenómenos son: los conflictos en el Parque Nacional Superagüi sobre mata atlántica que involucran a las comunidades de pescadores, en el estado de Paraná, Brasil; o bien los conflictos en los Parques Nacionales Naturales de Guacheneque o Los Nevados, etc, sobre los páramos y bosques altoandinos de Colombia, involucrando a comunidades indígenas y campesinas; o el caso del Bosque de Protección Alto Mayo, en Perú, donde se enfrentan guardianes ambientalistas y comuneros campesinos. En estas disputas colisionan incluso las legislaciones ambientales con aquellas regulaciones celebratorias de los derechos de las poblaciones tradicionales. La represión o expulsión (desalojos) de estas poblaciones de las áreas protegidas sigue siendo una opción posible en el abanico de los ejecutores de política pública. Estas poblaciones locales han sido denominadas por algunos investigadores y ONGs como "refugiados víctimas de la conservación" (REDD-MONITOR, 2009).

La gobernanza ambiental adquiere carácter dramático en los casos en que las poblaciones, sobre todo rurales, se ven avasalladas en sus territorios ante iniciativas tales como los Pagos por Servicios Ambientales (PSE) o la Reducción de las Emisiones de Deforestación y Degradación de los Bosques (REDD). Se observa en tales casos que la agenda de la cuestión ambiental ha sido colonizada por una tendencia global hacia el neoliberalismo, en donde el mercado define los esquemas de conservación que básicamente reflejarían la mercantilización de la naturaleza, su puesta en valor económico, frente a unas poblaciones indígenas o campesinas en resistencia.

Algunos autores han definido estos programas de conservación PSE/REDD, a partir de la creación de reservas naturales o de secuestro de carbono, como modalidades de "acaparamiento verde" (green grabbin), pues implican procesos de "apropiación de tierra y recursos para fines ambientales" (VIDAL, 2008). Un conjunto de casos de acaparamiento verde se combinan con cambios en el uso de suelo. Ejemplo son los cultivos de palma aceitera en Colombia, que son por un lado el resultado del desplazamiento de campesinos por grandes empresas, y por otro sustituyen el bosque nativo por este cultivo reconocido por el programa REDD como sumidero de carbono. 
Y Argentina no ha estado exenta de estos procesos. En la actualidad se registra una ascendente preocupación por parte de los diferentes poderes estatales respecto a la degradación de los ecosistemas y sus consecuencias sobre las poblaciones. Un indicador a nivel nacional de esta situación es la inclusión en la Constitución Nacional en 1994 (artículo 41 y 43) de una serie de garantías constitucionales en torno a la protección del medio ambiente, conservación de los así denominados "recursos naturales" y la responsabilidad por el daño ambiental; estableciendo a su vez, las atribuciones generales para la gestión ambiental, dividiéndolas entre el gobierno federal y los provinciales. Esto ha permitido a diferentes autores afirmar que el Derecho ambiental es mucho más que una disciplina autónoma del derecho; es el motor de cambio hacia una nueva cultura jurídica (CAFFERATA; 2004, BORRERO NAVIA; 2001). En efecto, Cafferata (2004) sostiene que a partir de la aparición del "paradigma ambiental" ha habido un cambio en las estructuras clásicas del derecho (por ejemplo, la acción judicial tiende deja de asumir un carácter reparatorio para ser anticipatoria, preventiva y de evitación del daño; el juez, deja su tradicional papel pasivo para transformarse en un magistrado activo, en un rol propio de la "justicia de acompañamiento"; conlleva cambios en la legitimación de obrar, la carga de la prueba, la apreciación de la prueba, los efectos de la sentencia, entre otras cuestiones.

En este contexto, se sanciona en el año 2002, "Ley General del Ambiente", la cual establece los presupuestos mínimos para el logro de una gestión sustentable y adecuada del ambiente, la preservación y protección de la diversidad biológica y la implementación del desarrollo sustentable, bajo el principio precautorio, el cual sostiene que "cuando haya peligro de daño grave o irreversible la ausencia de información o certeza científica no deberá utilizarse como razón para postergar la adopción de medidas eficaces, en función de los costos para impedir la degradación del medio ambiente" (art. 4). En base a ésta y bajo la égida de los "presupuestos mínimos" 5 se sancionaron un importante número de leyes ${ }^{6}$ para hacer frente al

\footnotetext{
${ }^{5}$ Por presupuestos mínimos se entiende normas de base sobre las cuales se va a construir el edificio total normativo de la tutela ambiental en la Argentina, de organización federal. Se trata de normas de aplicación en todo el territorio de la Nación, básicas, de un umbral, de un "piso inderogable".

${ }^{6}$ Ley 25.612 Presupuestos Mínimos para la Gestión Integral de los Residuos Industriales y de Actividades de Servicio; Ley 25.670 Presupuestos Mínimos para la Gestión y Eliminación de PCBs; Ley 25.675 Presupuestos Mínimos para la Gestión Sustentable y Adecuada del Ambiente; Ley 25.688 Régimen de Gestión Ambiental de Aguas; Ley 25.831 Información Pública Ambiental; Ley 25.916 Gestión de Residuos Domiciliarios; Ley 26.331 Presupuestos Mínimos de Protección Ambiental de los Bosques Nativos; Ley 26.562 Presupuestos Mínimos de Protección Ambiental para el Control de las
} 
impacto de diversas externalidades que la implementación de emprendimientos productivos y/o planes de infraestructura pueden ocasionar tanto sobre el medio natural como sobre la salud de las personas.

En el próximo apartado analizaremos tres dispositivos de regulación ambiental que si bien tienen por objetivo el aprovechamiento sustentable de los recursos y la reducción de los impactos negativos sobre el ambiente, conllevan a la apropiación y privatización de los bienes públicos de uso comunitario (bosques, ríos, agua y biodiversidad).

\section{Los dispositivos de gobernanza ambiental y la apropiación privada de la vida en Argentina}

Hemos seleccionado tres dispositivos o modalidades de gobernanza ambiental en Argentina: modelo silvopastoril para el aprovechamiento del bosque nativo, corredores biológicos para el aprovechamiento de la biodiversidad, y los estudios de impacto ambiental de la acción económica de gran escala sobre los modos de vida locales. Existen muchos otros que funcionan incluso de forma integrada con estos. Es el caso de los corredores biológicos que se articulan con las áreas protegidas, o el manejo del bosque nativo que se vincula con la sustentabilidad ambiental y social de las comunidades indígenas, etc.

Aprovechamiento sustentable del bosque: modelo silvopastoril

A fines del año 2007 se sancionó la ley nacional Nro. 26.331 de "Presupuestos Mínimos de Protección Ambiental de los Bosques Nativos", entre cuyos objetivos se encuentra promover la conservación mediante el Ordenamiento Territorial de los Bosques Nativos (OTBN) y regular la expansión de la frontera agropecuaria y de cualquier otro cambio de uso del suelo. EI OBTN, que debía ser aprobado en cada una de las provincias en el plazo de un año y a través de una consulta ciudadana, categoriza los usos posibles para las tierras boscosas: desde la conservación hasta la posibilidad de transformación para la agricultura, pasando por el uso sustentable del bosque ${ }^{7}$. En este marco de participación ciudadana, especialmente en las

Actividades de Quema; Ley 26.639 Régimen de Presupuestos Mínimos para la Preservación de los Glaciares y del Ambiente Periglacial

${ }^{7}$ EI OBTN zonifica los bosques de la siguiente manera: Categoría I (rojo): sectores de muy alto valor de conservación que no deben desmontarse ni utilizarse para la extracción de madera y que deben mantenerse como bosque para siempre. Incluirá las reservas naturales y sus áreas circundantes, que 
provincias con fuerte presencia de organizaciones campesinas e indígenas (como puede ser Salta, Chaco, Córdoba y Santiago del Estero) se generaron conflictos por la definición de los criterios a tener en cuenta en la construcción de la zonificación no sólo porque la Secretaria de Ambiente y Desarrollo Sustentable (SAyDS) no desarrollo guías ni instructivos que orienten la aplicación de los criterios de sustentabilidad que ordena la ley ${ }^{8}$ (AGN, 2013) sino también porque estos actores hacen visible el antagonismo de modos de manejo y significación de los bienes naturales, que se plasman en territorialidades diferentes y distintas referencias a la noción de sustentabilidad o conservación del monte o bosque nativo.

En este contexto, la implementación de la ley de bosques nativos en Argentina ha tenido una serie de dificultades en su implementación que conllevan a la apropiación y mercantilización de los mismos. En efecto, del informe generado por la Auditoría General de la Nación (2013) se destaca la falta de armonización de la política sobre bosques nativos establecida en la ley y las políticas de desarrollo vinculadas a la producción agropecuaria ${ }^{9}$ y el incumpliendo de la realización de audiencias públicas previas como requerimiento para la autorización de proyectos de desmonte. Por otro lado, la implementación de la ley de bosques no pudo reducir la tasa de desmontes para ampliar pasturas de ganadería empresarial y de la frontera agrícola con monoculturas de gran escala, básicamente soja, girasol, maíz y algodón y que además, del cambio de uso del suelo, disminuyen el acceso a las históricas áreas de pastoreo pertenecientes a campesinos y comunidades indígenas.

Además, la instrumentación del cambio de uso de suelo fue readaptada bajo parámetros de sustentabilidad definidos por un andamiaje técnico, caso del modelo agronómico silvopastoril. En un trabajo sobre la implementación de la ley de bosques en la provincia del Chaco (BARBETTA, 2018) hicimos eco de los reclamos de dos ONG's ambientalistas (Greenpeace y Centro Mandela), las cuales denunciaban que

\footnotetext{
tengan valores biológicos sobresalientes, y/o sitios que protejan cuencas hídricas de importancia (nacientes de ríos y arroyos); Categoría II (amarillo): sectores de alto o medio valor de conservación, que pueden estar degradados pero que si se los restaura pueden tener un valor alto de conservación. Estas áreas no pueden desmontarse, pero podrán ser sometidos a los siguientes usos: aprovechamiento sostenible, turismo, recolección e investigación científica; Categoría III (verde): sectores de bajo valor de conservación que pueden transformarse parcialmente o en su totalidad, con la previa realización de una Evaluación de Impacto Ambiental.

${ }^{8}$ Situación que se hace más palpable en la falta de coherencia entre las categorías de conservación en aquellas jurisdicciones que comparten ecorregiones.

${ }^{9}$ Los fines estratégicos del Plan Estratégico Agroalimentario y Agroindustrial 2010-2020 del Ministerio de Agricultura, Ganadería y Pesca de la Nación están en conflicto con los objetivos de la Ley 26.331, pudiendo representar una amenaza para la conservación de los bosques nativos.
} 
los aprovechamientos forestales (sistema silvopastoriles) lejos de perseguir la sustentabilidad de los bosques nativos, tienen por objetivo ampliar la frontera agrícola, ganadera y forestal, ya que resultan un desmonte encubierto o en etapas. Para el Centro Nelson Mandela, los sistemas silvopastoriles no respetan los tres principios básicos que garantizan su sustentabilidad y continuidad del bosque nativo: "Estos principios son de estabilidad, biodiversidad y renovabilidad, de tal manera que las actividades productivas deben ser amigables y compatibles con la conservación y el mejoramiento de las riquezas naturales y de las cualidades físicas y biológicas del ecosistema local. Cualquier tipo de producción que se encare (...) debe armonizar producciones sustentables, en lo biológico y en lo económico, manteniendo la estabilidad del ecosistema"10.

Por otra parte, las constantes denuncias por desmontes ilegales por parte de organizaciones campesinas y comunidades indígenas así como también de ambientalistas no debe ser visto meramente como un déficit de regulación estatal, generalmente atribuido a la falta de financiamiento de los organismos dedicados a controlar sino como parte de lo que Pegoraro (2016) llama delito económico organizado, es decir, "la organización empresaria delictiva dedicada a negocios ilegales -legales de una cierta complejidad política -jurídica con la necesaria participación de profesionales o expertos y de manera frecuente con instituciones y/o funcionarios estatales que produce una recompensa económica importante y que sus participantes son inmunes social- penalmente" (PEGOGARO, 2016, p. 18). Delitos que no sólo fortalece y reproduce formas de dominación y sometimiento de la agricultura empresarial sobre campesinos e indígenas sino también consolida viejas alianzas tras un modelo agropecuario productivo extractivista.

Aprovechamiento sustentable de la biodiversidad: corredores biológicos y áreas protegidas

Existe acuerdo en señalar que el concepto de "corredor" fue utilizado por primera vez con un sentido biológico ligado a la "dispersión de las especies", en el año de 1936 (SHAFER, 1990). Es en la década de 1970 cuando se consolida la propuesta de creación de corredores o conexión entre espacios naturales para la conservación de la biodiversidad, frente a la conservación de fragmentos aislados de hábitats y

\footnotetext{
${ }^{10}$ http://www.centromandela.com/?p=1969
} 
paisajes (YERENA, 2004). La propuesta de corredores biológicos pone el eje de la conservación en el restablecimiento de la conectividad entre hábitats y paisajes del mismo tipo de modo tal de evitar el aislamiento y luego extinción de poblaciones animales y vegetales. Mayormente los corredores biológicos se han diseñado para conectar áreas protegidas (públicas o privadas), y pueden asumir diversas escalas entre macro corredores regionales y pequeños corredores de conservación local en casos de ecosistemas muy dañados. En el año de 1992 las Naciones Unidas acuñan el término a partir de la Conferencia de las Naciones Unidas sobre Medio Ambiente y Desarrollo.

Por su parte Argentina cuenta con alguna experiencia en este sentido. Desde propuestas de conservación, proyectos de ley, y la efectivización de algunos corredores biológicos. El conjunto de iniciativas en Argentina adoptan en general la definición de "corredor biológico" elaborada por la Comisión Centroamericana de Ambiente y Desarrollo: "un espacio geográfico o una unidad de paisaje típicamente lineal, que proporciona conectividad entre paisajes, ecosistemas y hábitat, naturales o modificados, y asegura el mantenimiento de la diversidad biológica y de los procesos ecológicos y evolutivos" (CCAD, 2005). En efecto, en lo que respecta al cumplimiento del objetivo de protección de la diversidad biológica, los corredores biológicos son distinguidos como dispositivos excepcionales. A su vez, la definición se orienta a instrumentar, por la vía de la creación de corredores biológicos, el enfoque propuesto por el Convenio de Diversidad Biológica de Naciones Unidas ${ }^{11}$, al cual Argentina suscribe desde el año 1992. El Convenio considera especialmente tres aspectos: la conservación de la diversidad biológica, el uso sostenible de sus componentes y la participación justa y equitativa de los beneficios derivados del uso de los recursos genéticos. La conservación de biodiversidad aparece entonces como precondición esencial para las estrategias de desarrollo sustentable. De manera tal, la definición de corredor biológico presupone integrar el objetivo de la conservación con el de uso sustentable de la biodiversidad, en base a la idea de conectividad entre ecosistemas. En este sentido los corredores biológicos se inscriben dentro del giro epistémico, que se viene dando desde los principios de la modernización ecológica, donde el

\footnotetext{
11 "El Convenio sobre la Diversidad Biológica (CDB) es un tratado internacional jurídicamente vinculante con tres objetivos principales: la conservación de la diversidad biológica, la utilización sostenible de sus componentes y la participación justa y equitativa en los beneficios que se deriven de la utilización de los recursos genéticos. Su objetivo general es promover medidas que conduzcan a un futuro sostenible" (ONU https://www.un.org/es/events/biodiversityday/convention.shtml)
} 
crecimiento económico y el cuidado del ambiente no serían acciones incompatibles sino complementarias.

Ha habido intentos de legislar -en el nivel nacional- sobre corredores biológicos. En el año de 2017 se presentó al Congreso de la Nación un proyecto de ley sobre "Corredores Biológicos" en general. En este proyecto se señalaba la adscripción al carácter "inclusivo, participativo y descentralizado" para la implementación de corredores biológicos. El hincapié puesto en la cooperación de instituciones y sectores diferentes era destacado como "enfoque alternativo a las formas convencionales de la conservación de la diversidad biológica". Se buscaba vincular directamente a los corredores biológicos con el ascendente paradigma de la gobernanza ambiental.

Existen también iniciativas sobre ecosistemas específicos. Tal es el caso del corredor biológico del Rio Paraná (proyecto ley 9320-D-2014), del corredor biológico marítimo sobre el atlántico que proteja incluso ballenas, del corredor biológico Binacional Argentina-Chile para conservar la biodiversidad del bosque andino patagónico.

Algunas provincias argentinas ya cuentan con corredores biológicos. Misiones presenta el caso de mayor difusión: el corredor biológico Urugua-í - Foerster para recuperar el bosque (mata) atlántico o paranaense. Este corredor busca, desde el año 2002, dar conectividad a la fauna y flora del Parque provincial Urugua-í con el Parque provincial Guardaparque Horacio Foerster. Para ello se ha creado una trama geográfica basada en múltiples reservas privadas que atraviesa rutas y caminos con el sistema de "eco-ductos"12. La pretendida actitud "pionera" del gobierno de la provincia de Misiones en materia de corredores biológicos, que permitan conservar la diversidad de fauna y flora, parece complementarse con la promoción del aprovechamiento de esa biodiversidad con la Biofábrica Misiones SA. Esta empresa creada por el Estado se dedica a la "investigación, desarrollo, producción y comercialización de productos y servicios a base de procesos biotecnológicos”, para lo cual "almacena datos genéticos" (banco de datos genético: ley provincial 4.464) y alberga un laboratorio para la "propagación masiva de plantas in vitro, casas de cultivo y viveros". Se trata de una empresa estatal que articula con diversas instituciones públicas y privadas de ciencia y técnica: Instituto Nacional de Tecnología

\footnotetext{
${ }^{12}$ https://www.argentinaforestal.com/2019/07/25/corredor-biologico-urugua-i-foerster-logra-laregeneracion-del-bosque-altlantico-y-la-recuperacion-de-la-fauna-en-el-norte-de-misiones/
} 
Agropecuaria, Universidad Nacional de Misiones, Universidad Nacional del Nordeste, Universidad Nacional Marta Abreu de las Villas (Cuba), entre otros (Senado de la Nación 2015).

Existen corredores biológicos que pretenden articular agro-ecosistemas. Es el caso del corredor de conservación del arroyo Ayuí, en provincia de Entre Ríos. Esta iniciativa partió de una ONG (Fundación Hábitat y Desarrollo) conjuntamente con una empresa (Masisa Argentina) para la preservación de la vida silvestre y la "salud" del arroyo Ayuí, conectando predios de explotación forestal. El objetivo explicitado se refiere a dos cuestiones, como es la protección del cauce hídrico y a la vez a los beneficios por los servicios ambientales que trae. Según los impulsores, la conectividad lograda por el corredor eco-productivo permitiría "maximizar el uso sostenible del bosque y los beneficios derivados de los servicios ambientales". Cabe destacar en este caso que se define al corredor como un espacio diferenciado de un área protegida, puesto que "se busca es minimizar los efectos adversos de las actividades del hombre y promover un aprovechamiento sustentable de los recursos naturales" ${ }^{13}$.

Existen otros corredores biológicos en el país, ubicados en diferentes provincias como Salta, Santa Fe, Tucumán, etc. En todos ellos se destaca el favorecimiento de la movilidad de especies mediante la conectividad de espacioshabitats o áreas protegidas. Por lo general en estos casos el Estado aparece en articulación con sectores empresariales o del tercer sector. Es común también que la justificación para crear corredores sea en términos de la posibilidad de realizar estudios, monitoreos, inventarios, etc., mixturado con la puesta en valor de la biodiversidad o dicho también "aprovechamiento sustentable de los recursos naturales", y con la posibilidad de la sinergia colectiva dada la "participación" de todos los "interesados". Como lo expresara el director de la Fundación Hábitat y Desarrollo (ONG de Entre Ríos): "La conservación no tiene sentido con intentos individuales sino que es indispensable tener un 'colectivo', pensar en común" (APARICIO, 2015).

De este breve repaso destacamos que los corredores biológicos o ecológicos (incluidos los agro-ecológicos) se relacionan directamente con el control de la biodiversidad, un recurso natural que se ha vuelto estratégico. Diversidad biológica

\footnotetext{
${ }^{13}$ https://www.noticiasagropecuarias.com/2015/10/07/como-hacer-un-corredor-biologico-entrecampos-productivos/
} 
que se expresa en términos de germoplasma, genes del reino animal y vegetal, así como los conocimientos asociados a su detección y uso (DELGADO RAMOS, 2015). Por ello el acceso a la biodiversidad equivale al acceso a los recursos genéticos del planeta. Proceso que avanza en un contexto en el cual las tecnologías de punta del siglo XXI están directamente relacionadas con la conservación/apropiación/manejo de los recursos bióticos. En términos de la juridicidad global se habla ya del "derecho génico" (DELGADO RAMOS, 2015). Según Gian Carlo Delgado Ramos (2015) la biodiversidad tiene hoy un carácter geopolítico y económico central. La biodiversidad es objeto de políticas imperiales y disputas corporativas. En este contexto los corredores biológicos y las áreas protegidas forman parte de una disputa actual que se busca resolver con la creación de un sistema de bioprospección mundial y un sistema mundial de derechos de propiedad intelectual (DELGADO RAMOS, 2015).

El estudio de impacto ambiental

En el apartado anterior marcábamos la importancia del derecho ambiental como motor de cambio de una nueva cultura jurídica anclado en el principio precautorio. Éste opera sobre el riesgo que trae el desarrollo, haciendo que el derecho ambiental sea un ámbito fértil para la regulación de la incertidumbre, la prevención del daño pero también para su restauración, recomposición o compensación (CAFFERATA, 2004). En este contexto, el Estudio de Impacto Ambiental (EIA) es el proceso que permite identificar, predecir, evaluar y mitigar los potenciales impactos que un proyecto de obra o actividad puede causar al ambiente, en el corto, mediano y largo plazo; previo a la toma de decisión sobre la ejecución de un proyecto. Es un procedimiento técnico-administrativo previsto en la Ley № 25.675 General del Ambiente con carácter preventivo, que permite una toma de decisión informada por parte de la autoridad ambiental competente respecto de la viabilidad ambiental de un proyecto y su gestión ambiental. Pero además, es utilizado dentro del campo jurídico como forma de prueba para la resolución de aquellos litigios que podríamos enmarcar dentro de lo que la ecología política ha definido como disputas por externalidades negativas, por lo desproporcionado o desigual de los costos del impacto ambiental, pero que también ha relacionado con las luchas por la injusta cantidad de recursos naturales que se apropian y consumen determinados sectores sociales o países (MARTÍNEZ ALIER, 2006). 
Según Berros (2010), el procedimiento de evaluación de impacto ambiental es un dispositivo jurídico que da cuenta de la emergencia de la racionalidad precautoria que ensambla "lo científico" y "lo político" para la toma de decisiones en relación con los riesgos. Si consideramos que se aplica el principio precautorio cuando haya peligro de daño grave o irreversible y ausencia de información o certeza científica para impedir la degradación del medio ambiente, el estudio de impacto ambiental viene a reintroducir cierto criterio de certeza en base a postulados científicos que intentan dar respuesta a la pregunta acerca de cómo vincular riesgos posibles con un determinado agente contaminante. En otras palabras, se recurre a un nuevo campo social, el del conocimiento científico, para dirimir acerca de la idea de daño o la plausibilidad del riesgo. Con ello, existe un deslizamiento del daño como riesgo aceptable para los actores del Estado y del mercado, que comparten en ese sentido la escisión en el par sociedad-naturaleza desde el andamiaje científico y las reglas del mercado. De modo tal se construye al ambiente por fuera de las relaciones sociales, como objeto (la naturaleza) desentrañable, predecible y medible en sus relaciones internas y en los posibles impactos humanos. Pero además, permite tanto los actores gubernamentales como como a los actores del mercado envestirse de un discurso eco-eficiente sobre la compatibilidad entre la producción de bienes y la sustentabilidad ecológica (MARTINEZ ALIER, 2004), respaldado en una ciencia y una juridicidad gerencial del ambiente (evaluación de riesgo, remediación de daño, economización de la ecología, etc).

Sin embargo, volviendo a la idea de que la capacidad de acción de la ciencia, no fue acompañada de forma similar por su capacidad de predicción (SANTOS, 2009), el principio precautorio, la indeterminación causal o el grado de certeza científica alcanzado no es condición suficiente para que las poblaciones no se sientan lesionadas. En efecto, mientras que la plausibilidad del riesgo es evaluado al interior del campo científico, la idea de daño se construye en el campo social y político. En efecto, éste es percibido por los sujetos afectados y se construye a partir de determinar tanto las causas y sus causantes a partir de conocimientos que la ciencia moderna ha descalificado históricamente, como ser las evidencias de la experiencia inmediata y la sabiduría práctica y cotidiana de los hombres.

En efecto, en Argentina podemos nombrar algunos casos paradigmáticos que reflejan dicha situación. Uno de ellos fue en conflicto binacional entre Uruguay y 
Argentina surgido en la ciudad de Gualeguaychú a raíz de la decisión del gobierno de Uruguay de habilitar la instalación de dos plantas de celulosa en Fray Bentos. el conflicto motivó la convergencia amplia de actores sociales de la ciudad de Gualeguaychú, agrupados bajo la consigna "No a las papeleras. Si a la vida", en una instancia de estructura asamblearia, que se asumía identitariamente como "ciudadana" y "ambiental" y que congregaba una diversidad de sujetos sociales: vecinos, comerciantes, docentes, agrupaciones ambientales y políticas, juveniles, etc. Los habitantes se envistieron de argumentos ecologistas como disposición para la lucha, que adquirió formas tales como la acción directa, la deliberación democrática, y el despliegue territorial. En estos términos la población confrontó con el gobierno de Uruguay y el argentino, por sus actitudes complacientes o indiferentes a la problemática ambiental que tal instalación de la industria desataría.

La preocupación principal parecía circunscribirse a los efectos nocivos sobre el agua del rio común y sobre el aire, que las Papeleras provocarían sobre las poblaciones aledañas, afectando las pautas de vida características de la ciudad. Es decir, el conflicto desde un inicio cuestionó la distribución injusta y perjudicial de los componentes contaminantes de las plantas de celulosa. Por su parte, la aparición de un discurso sobre la sustentabilidad estuvo orientado, no a la conservación de bienes naturales ligados a formas de reproducción social alternativas, sino a la defensa de un espacio social escencializado (la sociedad de Gualeguaychu) puesto en riesgo por un emprendimiento foráneo.

Destaquemos que en este conflicto fue ocupando un lugar central el debate en torno de las evaluaciones técnicas sobre los riesgos y los impactos, con informes y contra informes, que buscan construir impugnaciones de base científica en todos los casos. Todos los actores involucrados en este caso asumieron el conocimiento científico como incuestionable. De modo ha sido el conocimiento científico el lenguaje de valoración que se tornó sistema a ser aplicado para su resolución, y las diferentes posiciones disputaron al interior de ese mismo sistema de valoración. En este contexto, el 20 de abril de 2010 se dio a conocer el veredicto de la Corte Internacional de Justicia (CIJ) de La Haya, dependiente de la ONU, acerca de la demanda que Argentina inició contra Uruguay en mayo de 2006. El estudio de impacto ambiental determinó que no se habían encontrado "pruebas concluyentes" de contaminación general -salvo hechos aislados- que permitan establecer que la papelera esté 
"afectando la calidad de las aguas" del río por efecto de los efluentes que despide la planta papelera de la finlandesa Botnia. Sin embargo, más allá de la verdad científica construida, un asambleísta sostenía que "...no hay nada que demostrar, basta saber lo que es Botnia para saber que va a contaminar (...) Un técnico, un científico, una persona bien informada, pero no puede dudar jamás de que esta planta es altamente contaminante" (citado en GRAÑA, 2006: 76).

Un segundo caso de la utilización del estudio de impacto ambiental en el marco de un litigio ambiental que adquirió relevancia política y social no sólo de carácter provincial sino también nacional. En él se conjuraron la vulneración de los derechos indígenas con la disputa en torno a las formas de uso y manejo del bosque nativo en la provincia de Chaco. En el proceso judicial se pusieron en disputa dos órdenes de cosas íntimamente relacionados: las nociones de sustentabilidad presentes en cada uno de los sujetos intervinientes y las formas de uso, manejo y apropiación del bosque nativo. Si bien la sentencia habilitó la tutela activa de los bosques nativos y del derecho a un ambiente sano fundamentada a partir de los principios de sustentabilidad, de prevención y precautorio también implicó una reorientación de la demanda hacia el control de prácticas productivas que tienen por objetivo la utilización del bosque nativo en base a una racionalidad que pretende armonizar la explotación del mismo, en tanto recurso natural con la idea de desarrollo, a partir de la "extracción sustentable". Sin embargo para las comunidades indígenas no se trata exclusivamente de una disputa por las externalidades negativas producto una de una determinada forma de uso, manejo y apropiación del bosque nativo, sino que también se trata de la reivindicación de los derechos del ser en base a una noción de sustentabilidad que remite a la necesaria coexistencia entre un singular modo de vida y un determinado espacio geográfico. En este contexto, el reclamo indígena escenifica, por un lado, en el espacio público un debate en torno a la sustentabilidad a partir de la desmercantilización de los bienes comunes y el cuestionamiento de las políticas de desarrollo. Por el otro, en el campo jurídico, pone en discusión la función simbólica del juez en tanto que, si bien el paradigma ambiental implica un cambio de habitus, éste sigue adquiriendo un carácter eminentemente regulatorio, en nuestro caso, de las prácticas sociales que se dan en el espacio de la producción (BARBETTA, 2018).

Un tercer caso de relevancia para la Argentina es la apropiación y privatización del agua por parte de la megaminería. En los últimos años se destaca un fuerte 
activismo político contra los emprendimientos mineros que va desde la acción colectiva directa para frenar nuevos emprendimientos como presentaciones judiciales en contra de las externalidades o la apropiación privada del agua por parte de las actividades mineras. En este último caso, la justicia se limitó a recomendar a los gobiernos provinciales la realización de revisiones a los informes de impacto ambiental o que intervinieran en el cuidado del medioambiente en las zonas de explotación (BOTTARO, LATTA Y SOLA, 2004). Sin embargo, frente a las recomendaciones de una mayor o mejor regulación, el vínculo 'agua-vida' que expresa la valoración de los actores movilizados, en zonas donde un escenario de agudización de la escasez del agua, representa la imposibilidad de existencia de los proyectos de vida de las comunidades (BOTTARO, LATTA Y SOLA, 2004).

\section{Conclusiones: cuestión ambiental y territorios de vida}

En este trabajo analizamos la cuestión ambiental en un contexto caracterizado como de crisis del paradigma de la modernidad en el cual la regulación y la emancipación social se han visto colonizadas por la lógica de la apropiación y violencia. En este contexto de transición de la modernidad, la relación sociedad naturaleza ha sido resignificada en clave de crisis ecológica. Frente a este escenario, el arte neoliberal de gobierno ha propuesto como salida la gobernanza ambiental, en un intento por rehabilitar la regulación social. De este modo, la modernidad estaría encontrando, desde su propia lógica, soluciones a los problemas que ella mismo generó a partir de la fe irrestricta en torno al progreso científico y por ende, con la profundización de la naturaleza como artefacto. El éxito de este principio de autorregulación sería la modernización ecológica. Para ello se vale de un conjunto de dispositivos de gobernanza ambiental, entre los cuales están modelos sustentables de cambio de uso del suelo, conservación de la biodiversidad para la bioprospección, estudios de las externalidades ambientales de las actividades económicas (agroforestales, pasteras, mineras).

Sin embargo, del análisis realizado tanto a escala internacional como local de dichos dispositivos, sostenemos que la gobernanza ambiental, parafraseando a Boaventura de Sousa Santos, no regula ni emancipa sino que apropia y violenta. La dialéctica de la apropiación y/o violencia que registramos en el análisis de los casos de gobernanza ambiental en Argentina instrumentan la privatización y mercantilización 
de la naturaleza. En el caso de los estudios de impacto ambiental, la privatización estaría dada por la desigual apropiación de los procesos o dinámicas eco -sistémicas y de las cargas de contaminación (en el caso de la pastera y las mineras) o por la apropiación desigual del stock de recursos naturales (en el caso de bosque nativo). El caso de los corredores biológicos nos brinda otro aspecto de la privatización la cual supone la apropiación y su posterior, mercantilización, de los activos genéticos de esos territorios. Esta modalidad de gobernanza está asociada directamente con el funcionamiento de las áreas protegidas (para brindarles conectividad ecológica), que si bien no analizamos, vienen demostrando recientemente la articulación entre apropiación y situaciones extremas de violencia estatal caso de los desalojos de familias isleñas en el Parque Nacional Islas de Santa Fe (año 2019), o el asesinato de Rafael Nahuel y los heridos de la comunidad mapuche Lafken Winkul Mapu, en el Parque Nacional Nahuel Huapi (año 2017). El análisis de los manejos silvo-pastoriles, en el marco de la implementación de la ley de bosques, muestra que la privatización y la mercantilización se da, por un lado, a partir de una noción de sustentabilidad que acepta la modificación de los procesos productivos para reducir el impacto ambiental, considerando que la conservación es necesaria para el crecimiento económico. Por el otro, a través de la apropiación (muchas veces violenta, con asesinatos) de áreas de pastoreo históricas pertenecientes a campesinos y comunidades indígenas.

Frente a esta situación, campesinos, indígenas y poblaciones urbanas emergen en el espacio público planteando sus derechos. Unos, en base a un lenguaje de valoración ancestral y portando una racionalidad ambiental tal cual la entiende Enrique Leff (2004), otros a partir de los clivajes propios de la justicia ambiental. Sin embargo, todos ellos convergiendo en la defensa del espacio vital, ya sea en términos del uso comunitario de los bienes naturales o ya sea resistiendo a la desigual distribución de los daños ambientales. De este modo, confrontan la privatización y mercantilización propia de la gobernanza ambiental con los territorios de vida de las poblaciones locales.

\section{Referencias}

APARICIO, Gustavo (2015). Cómo hacer un corredor biológico entre campos productivos. Nota publicada en: https://www.noticiasagropecuarias.com/2015/10/07/ como-hacer-un-corredor-biologico-entre-campos-productivos/ 
ARGENTINA. AUDITORIA GENERAL DE LA NACION (AGN), AUDITORÍA DE GESTIÓN AMBIENTAL: LEY DE PROTECCIÓN DE BOSQUES NATIVOS, Disponible en: https://www.agn.gov.ar/informes/auditoria-de-gestion-ambiental- leyde-proteccion-de-bosques-nativos. Acceso: 25 de julio 2019

ARGENTINA. Senado de la Nación "Día Internacional de la Diversidad Biológica". Resolución 55/201 del Senado de la Nación Secretaria Parlamentaria Dirección General de Publicaciones (S-1604/15). Año del Bicentenario del Congreso de los Pueblos Libres", 2015. Acceso: 25 de julio 2019.

BARBETTA, Pablo Disputas por la significación del Bosque Nativo en la provincia de Chaco: daño, sustentabilidad y justicia ambiental Revista Electrónica. Instituto de Investigaciones Ambrosio L. Gioja, Número 21, pp. 1-24, Buenos Aires, Argentina diciembre 2018-mayo 2019.

BARBETTA, Pablo. COX DE BRITTO Monica, DOMÍNGUEZ Diego y PESSOA Kaue (2018). Neoliberalismo y cuestión ambiental: entre la crisis ecológica y la ruptura paradigmática". Entramados y Perspectivas, Revista de la Fac. de Cs. Sociales-UBA. $\left(\mathrm{N}^{\circ} 8\right)$ p. $333-359$. Buenos Aires,.

BARTRA, Armando. El hombre de hierro; límites sociales y naturales del capital. México: Editorial Itaca, UAM-UACM, 2008.

BERROS, María Valeria. Evaluación de impacto ambiental, una mirada como dispositivo jurídico de gestión de riesgos. Revista Derecho y Ciencias Sociales №2. Instituto de Cultura Jurídica y Maestría en Sociología Jurídica. FCJyS.UNLP, p. 68-83, 2010.

BORRERO NAVIA, José María. "Derecho ambiental y cultura legal en América Latina" In LEFF, Enrique (coord.) Justicia ambiental: construcción y defensa de los nuevos derechos ambientales culturales y colectivos en América Latina, México: Programa de las Naciones Unidas para el Medio Ambiente y Red de Formación Ambiental para América Latina y el Caribe, 2001, p. 35-43.

BOTTARO, Lorena; LATTA, Alex; SOLA, Marian. La politización del agua en los conflictos por la megaminería:Discursos y resistencias en Chile y Argentina European Review of Latin American and Caribbean Studies Revista Europea de Estudios Latinoamericanos y del Caribe, issue 97, pp. 97 - 115, oct. 2017

CAFFERATA, Néstor. Introducción al derecho ambiental, México: INECC, 2004 Comisión Centroamericana de Ambiente y Desarrollo (CCAD) Corredor Biológico Mesoamericano. Disponible en: https://www.sica.int/documentos/corredor-biologicomesoamericano_1_7721.html. Acceso: 01 de agosto 2019

DE LISIO, Antonio. IIRSA: mucho de pasado desarrollista y poco de futuro sustentable. 2013 En https://www.vocesenelfenix.com/content/iirsa-mucho-depasado-desarrollista-y-poco-de-futuro-sustentable. Acceso: 21 de septiembre 2019

DELGADO RAMOS, Gian Carlo (2015). Biodiversidad, desarrollo sustentable y militarización : esquemas de saqueo en Mesoamérica. Mexico: Centro de 
Investigaciones Interdisciplinarias en Ciencias y Humanidades Universidad Nacional Autónoma de México, 2015.

GRAÑA, François. "No hay nada que demostrar, Botnia va a contaminar”. Un análisis del discurso de la Asamblea Ciudadana Ambiental de Gualeguaychú Revista de Ciencias Sociales, núm. 26, Universidad de la República Montevideo, Uruguay pp. 73-83, abril, 2010

LEFF, Enrique. La Geopolítica de la Biodiversidad y el Desarrollo Sustentable: economización del mundo, racionalidad ambiental y reapropiación social de la naturaleza", In AA.VV. Semináro Internacional REG GEN: Alternativas Globalização, Rio de Janeiro: UNESCO, 2005. Disponible en: http://bibliotecavirtual.clacso.org.ar/ar/libros/reggen/pp12.pdf

LEFF, Enrique. Racionalidad Ambiental. La reapropiación social de la naturaleza, México: Siglo XXI, 2004.

MARTÍNEZ ALIER, J. Los conflictos ecológico-distributivos y los indicadores de sustentabilidad. Polis. Revista Latinoamericana, n. 13. P. 1-15, agosto, 2006.

MARTÍNEZ ALIER, Joan. El ecologismo de los pobres. Conflictos ambientales y lenguajes de valoración. Barcelona: ICARIA, 2004.

MARX, Karl. El Capital. Libro I. Buenos Aires: Siglo XXI, 1997.

O'CONNOR, James. ¿Es Possible El Capitalismo Sostenible? in H. Alimonda (Org.). Ecología política. Naturaleza, sociedad y utopia, Buenos Aires: Clacso, 2002.

PEGORARO, Juan. Los lazos sociales del delito económico y el orden social, Buenos Aires: EUDEBA, 2016.

POLANYI, Karl. La Gran Transformación. Crítica del liberalismo económico Madrid: La Piqueta, 1989.

SANTOS, Boaventura de Sousa. Sociología jurídica crítica. Para un nuevo sentido común en el derecho. Bogotá: Instituto Latinoamericano de Servicios Legales Alternativos (ILSA), 2009.

SANTOS, Boaventura de Sousa. Crítica de la razón indolente. Contra el desperdicio de la experiencia, España: Editorial Desclée de Brouwer, 2000.

SANTOS, Boaventura de Sousa. Reiventar la democracia, reinventar el Estado, Buenos Aires: CLACSO, 2006.

SANTOS, Boaventura de Sousa. y RODRÍGUEZ GARAVITO, César. El derecho y la globalización desde abajo. Hacia una legalidad cosmopolita. Barcelona: Anthropos, 2007.

SEOANE, José. 2017. Las (Re) Configuraciones Neoliberales Ambiental: Una Arqueología de Los Documentos de Naciones Unidas Sobre El Ambiente 1972 2012. Buenos Aires: Luxemburg, IEALC, GEAL, 2017. 
SHAFER, C. Nature reserves: island theory and conservation practice. Smithsonian Institution Press, Washington, D.C, 1990.

VIDAL, John. The great green land grab, in The Guardian, febrero 2008, Londres, URL: www.theguardian.com/ environment/2008/feb/13/conservation. Acceso: 27 de septiembre 2019.

YERENA, Edgard (2004). Corredores: ¿de qué estamos hablando? Documento base para el foro electrónico sobre corredores y enfoque ecosistémico. Departamento de Estudios Ambientales Universidad Simón Bolívar. Caracas, Venezuela, 2004. 